Oncology

Research and

Treatment

\title{
ASCO Annual Meeting 2020: Nierenzellkarzinom - weiterhin immunbasierte Kombinationstherapien bei Patienten mit günstiger Prognose?
}

Die Therapie des Nierenzellkarzinoms (RCC) hat sich in den letzten Jahren durch den Einzug der (kombinierten) Immuntherapie - besonders in der Erstlinie stark verändert. Derzeit stehen laut der aktuellen Empfehlungen der European Association of Urology (EAU) für Patienten mit einem klarzelligen (cc) RCC mit günstiger Prognose als Standardbehandlung die Kombination aus dem PD-1-Inhibitor Pembrolizumab und dem gegen den VEGF-Rezeptor gerichteten Tyrosinkinase-Inhibitor (TKI) Axitinib zur Verfügung [1]. Sowohl diese Kombinationen als auch die aus dem PD-1-Inhibitor Nivolumab und dem CTL-4-Inhibitor Ipilimumab werden bei Patienten mit intermediärer oder ungünstiger Prognose in der europäischen Leitlinie empfohlen.

Die Einteilung der Risikogruppen basiert auf dem IMDC-Score, der auf dem Performance-Status eines Patienten, Laboruntersuchungen sowie der Zeit zwischen Diagnose und Beginn einer Systemtherapie beruht [2].

\section{KEYNOTE-426: Update zur Kombinationstherapie} beim fortgeschrittenen RCC je nach Risikogruppe

Die Ergebnisse der ersten Interimsanalyse der randomisierten, multizentrischen, offenen, aktiv-kontrollierten KEYNOTE-426-Studie mit 861 ccRCC-Patienten führten zur Zulassung der Kombination Pembrolizumab plus Axitinib für die Erstlinientherapie des fortgeschrittenen RCC $[3,4]$. Beeindruckend war besonders das signifikant verlängerte Gesamtüberleben (OS) mit der Kombination im Vergleich zu Sunitinib (Hazard Ratio [HR] (95\% Konfidenzintervall $[\mathrm{KI}]): 0,53[0,38-0,74] ; \mathrm{p}=0,00005)$ sowie das signifikant verlängerte progressionsfreie Überleben (PFS) von 15,1 Monaten mit Pembrolizumab plus Axitinib vs. 11,0 Monaten mit Sunitinib (HR [95\% KI]: 0,69 $[0,56-0,84] ; \mathrm{p}=0,00012)$. Die objektive Ansprechrate lag bei 59,3\% für die Kombination und 35,7\% für Sunitinib $(p<0,0001)[3,4]$.

Auf dem diesjährigen Kongress stellte das Forschungsteam um Elisabeth Plimack nun eine aktualisierte Analyse der KEYNOTE-426-Studie nach verlängertem Followup vor [5]. Im Vergleich zur damaligen medianen Nachbeobachtungszeit von 12,8 Monaten, betrug sie in der aktualisierten Auswertung mindestens 23 Monate. Die aktualisierte HR für das OS lag nun bei 0,68 (95\% KI: $0,55-0,85 ; \mathrm{p}<0,001$ ) im Vergleich zu Sunitinib. Die Kombinationstherapie verlängerte auch nach dem Update das PFS signifikant auf 15,4 Monate im Vergleich zu 11,1 Monaten unter Sunitinib und reduzierte so das Risiko für eine Progression um 29\% (HR: [95\% KI]: 0,71 [0,60$0,84] ; \mathrm{p}<0,0001)$. Allerdings profitierten nicht alle untersuchten Subgruppen. Während sich bei der Analyse der Patientengruppe mit günstigem Risikoprofil weder beim OS noch beim PFS ein signifikanter Unterschied zwischen den Behandlungsarmen zeigte, verbesserte die CITKI-Kombination sowohl das Gesamt- als auch das progressionsfreie Überleben signifikant für Patienten mit intermediärer oder ungünstiger Prognose [5]. 
Die Analyse der verlängerten Nachbeobachtungszeit bestätigte damit insgesamt die Wirksamkeit im Vergleich zu Sunitinib beim ccRCC. Die Ergebnisse der Subgruppenanalyse könnten aber in Zukunft zur Diskussionen im Therapiealltag in der Gruppe der Patienten mit günstiger Prognose bezüglich des Einsatzes von Pembrolizumab plus Axitinib führen.

\section{Progress nach immunbasierter Therapie beim metastasierten ccRCC - weitere Kombination?}

Checkpoint-Inhibitor-basierte Kombinationstherapien sind der neue Standard in der Erstlinienbehandlung beim RCC [1]. Das hat auch Auswirkungen auf Folgetherapien und bisher liegen wenige Daten zur Behandlung bei einem Krankheitsprogress nach Behandlung mit CI wie PD-1- oder PD-L1-Antikörpern vor. Nun präsentierten Chung-Han Lee und Kollegen eine multizentrische, offene Phase-II-Studie mit 104 ccRCC-Patienten, die mit einer Kombination aus Pembrolizumab und dem TKI Lenvatinib nach einem Progress unter vorheriger
PD-1/PD-L1-Checkpoint-Therapie behandelt wurden [6]. Genauer hatten zuvor 65 Patienten eine Kombination aus Anti-PD-1/PD-L1 plus Anti-VEGF sowie 37 Patienten Nivolumab plus Ipilimumab erhalten. Die Ansprechrate von Pembrolizumab plus Lenvatinib lag bei 55\% und die mediane Ansprechdauer bei 12 Monaten. Nach einem Behandlungsjahr waren 77\% der Patienten noch am Leben und $44 \%$ zeigten bis dahin keinen Krankheitsprogress [6].

Die Kombination Pembrolizumab plus Lenvatinib wies das zu erwartende Nebenwirkungsspektrum und eine vielversprechende Anti-Tumoraktivität nach Versagen vorheriger Immuntherapien mit PD-1/PD-L1-Inhibitoren auf. Die Ergebnisse liefern erste Antworten auf die Frage, wie nach einem Progress unter einer immunbasierten Erstlinientherapie zukünftig therapeutisch fortgefahren werden könnte. Dabei zeigte sich, dass in der Subgruppe der mit einer Kombination aus CI plus TKI behandelten Patienten eine Wiederholung dieses Ansatzes mit anderen Wirkstoffen (CI plus TKI) in der Folgelinie ebenfalls wirksam sein kann.

Gerhard Emrich, Berlin

\section{Literatur}

1 Ljungberg B. EAU Guideline Renal Cell Carcinoma. 2020; Available from: https://uroweb. org/guideline/renal-cell-carcinoma/ (Aufgerufen: 18.06.2020).

2 Heng DYC, et al. External validation and comparison with other models of the International Metastatic Renal-Cell Carcinoma Database Consortium prognostic model: a population-based study. The Lancet Oncology, 2013. 14(2): 141-148.
3 MSD, Fachinformation KEYTRUDA. März 2020.

4 Rini BI, et al. Pembrolizumab plus Axitinib versus Sunitinib for Advanced Renal-Cell Carcinoma. N Engl J Med, 2019. 380(12):1116-1127.

5 Plimack ER. Pembrolizumab plus Axitinib versus Sunitinib as First-Line Therapy for Advanced Renal Cell Carcinoma: Update Analysis of KEYNOTE-426. ASCO Annual Meeting 2020 Virtual, Abstract 5001.
6 Lee $\mathrm{C}-\mathrm{H}$, et al. Phase 2 trial of lenvatinib plus pembrolizumab for disease progression after PD-1/PD-L1 immune checkpoint inhibitor (ICI) in metastatic clear cell renal cell carcinoma. ASCO Anual Meeting 2020 Virtual, Abstract 5008. 